\title{
Ergonomic Characteristics of Gestures for Front- and Back-of-Tablets Interaction with Grasping Hands
}

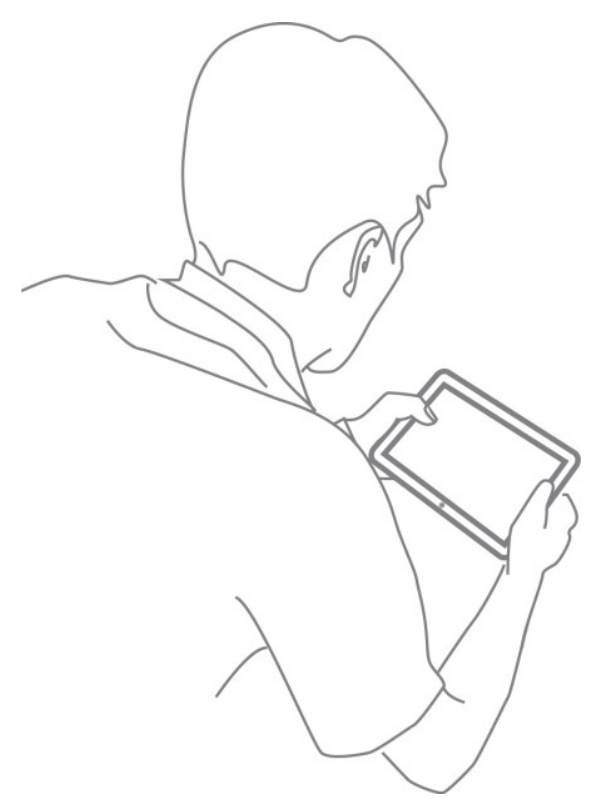

Figure 1. Hand configuration for holding the device while filling a form (left) and touch heatmaps (right).

\section{Katrin Wolf}

University of Stuttgart

katrin.wolf@acm.org

Robert Schleicher

Technical University Berlin

robert.schleicher@tu-berlin.de

\section{Michael Rohs}

Leibnitz University Hannover

michael.rohs@hci.uni-hannover.de

\begin{abstract}
The thumb and the fingers have different flexibility, and thus, gestures performed on the back of a held tablet are suggested to be different from ones performed on the touchscreen with the thumb of grasping hands.

APIs for back-of-device gesture detection should consider that difference. In a user study, we recorded vectors for the four most common touch gestures. We found that drag, swipe, and press gestures are significantly differently when executed on the back versus on the front side of a held tablet. Corresponding values are provided that may be used to define gesture detection thresholds for back-of-tablet interaction.
\end{abstract}

\section{Author Keywords}

Gesture; Ergonomics; Tablet; Touch; Back-of-Device.

\section{ACM Classification Keywords}

H.5.2 [Information inter-faces and presentation]: User Interfaces - Ergonomics; Haptic I/O.

\section{Introduction}

Tablet interaction with grasping hands is, similar to one-handed phone interaction, (A) a common tablet grip that (B) constrains the gesture feasibility.(A),

shown in Figure 1, has been stated by Wagner et al. [8] 
as well as by Oulasvirta et al. [6]. That grip is ergonomic and allows for holding tablet stable because both hands are holding the device, which reduces the gesture feasibility at the same time. Enabling interaction on the back of the device may compensate the reduced interaction design space. The question targeted here is: How do typical touch gestures look like if executed with grasping hands on the front as well as on the back of the device?

\section{Related work}

Hoggan et al. [3] investigated the factors effecting touch gesture performance and ergonomics.

Parameters of a rotation gesture were systematically controlled in the experiment, such as rotation angle, rotation direction, distance between fingers, and position. The study shows that within-gesture parameters, such as the angle of the fingers (given as start and final position of the two touch points) influence the gesture execution time. Wobbrock et al. [10] compared the performance of the index finger and the thumb for front- and back-of-phone interactions and showed that with shorter interaction times, when using the thumb on the front screen, this interaction technique has greater ergonomic values than e.g. interacting with the thumb on the rear of the phone. A comparison of front and back touch with transparent double sided touch displays, inspired by Hiraoka et al. [2] and Wigdor et al. [9], showed that touch performance on the back is lower in terms of target acquisition time, while accuracy was equal [5], as well as the position-dependent reachability [11]. However, some work on gesture ergonomics and comparison of front and back touch was done; no vector comparison for both front- and the back-of-tablet gesture interaction was done.

\section{Method}

Design. An $4 \times 2 \times 5 \times 2$ within design with repeated measurements experiment was designed where 18 participants, (7 female, mean Age $=28, \mathrm{SD}=3$ ) volunteered. The dependent variables were gesture, direction, digit, hand, and device side. The independent variables were $x$-positions and $y$-positions of the changing touch points while gestures execution.

Apparatus \& measurements. An application running on an ASUS Eee Pad Transformer TF101 was developed for recording the touch events. For the back-of-device conditions, the device was flipped so that the back side was facing the users, while the touchscreen allowed for recording touch events on the back. The touch events were recorded in logfiles and contained per participant five trials per device side, hand and digit for four gestures, 2 gesture orientations (horizontal, vertical) for swipe and drag; 10800 gestures in total.

Handedness and demographic data were recorded by a questionnaire.

Task\& procedure. The participants were asked to execute tap, press, drag, and swipe gestures with both hands and each digit per hand without getting system feedback. The drag and swipe gesture were executed in the horizontal as well as in the vertical direction. After an introduction, the participants solved the gesture execution task five times with each finger per hand. Half of the participants started with their dominant, the other half with their non-dominant hand in a standing position. All combinations were executed separately on the front and the back of the device five times each while holding the device with two hands in a standing position. After completing the tasks, the participants filled in a questionnaire. 

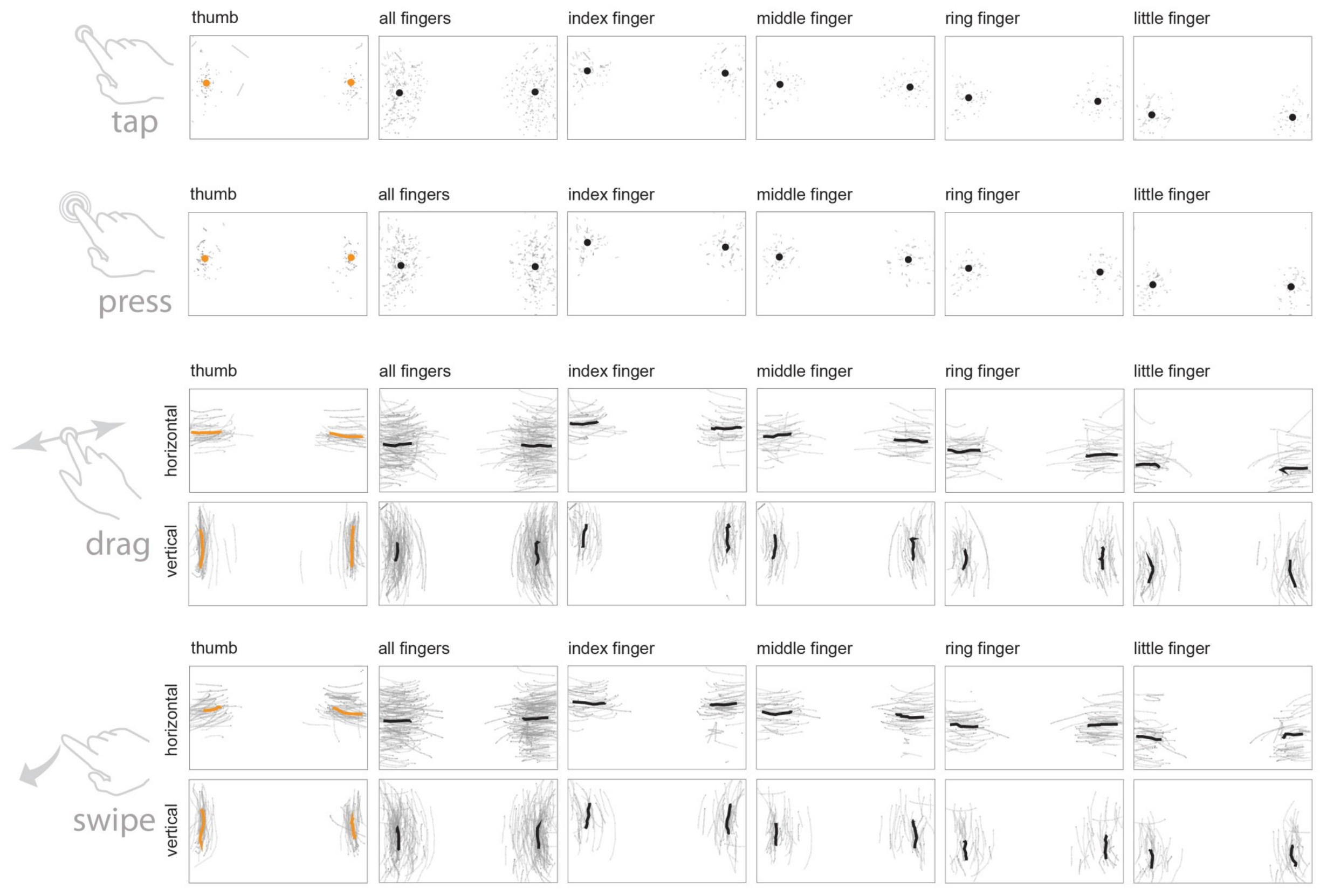

Figure 2. Gesture vectors (grey) and median for front (orange) and back (black, see-through view) of a tablet, drawn to scale of a tablet screen of $1280 x 742$ pixels. 


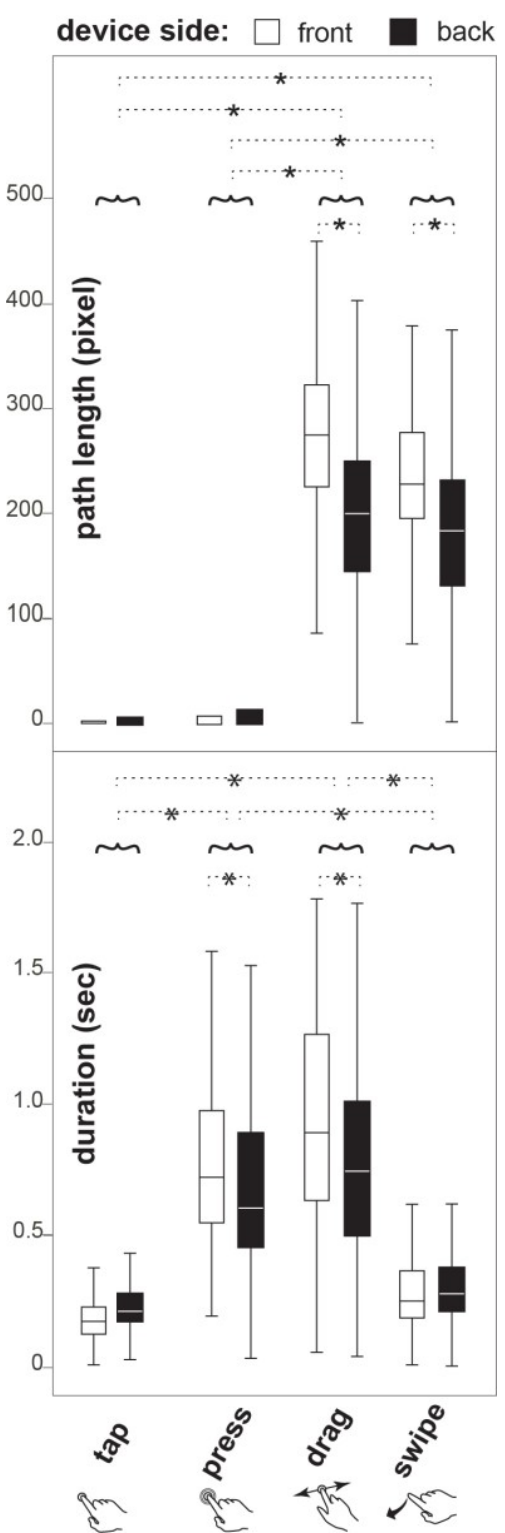

Figure 3. Boxplot of path length \& duration for each gesture and significant differences* in between.

\section{Results}

For the gesture parameters (touch position, path length, and execution duration), we tested whether they are affected by gesture orientation or by the device side (front versus back of tablets) as well as whether they vary between the dominant or nondominant hand. The individual vectors for each gesture tap, press, drag, and swipe, and typical gesture vectors using the median (see Table 1) are drawn (in orange and in black) to scale of a tablet screen of $1280 \times 742$ pixels in Figure 2. The individual gesture vectors differ a lot, which is shown by the widespread location of the whole set of gesture vectors that are drawn in gray.

Gesture position. Regarding the gesture positions, the median values for tap and press gestures were always located close to the vertical edges where that hands are places. The gesture positions differ a lot in their vertical position but less horizontally. The touch positions of the gestures when executed on the front are again slightly closer to the edge/hand palm than those performed at the back of the device.

Gesture shape. Different gesture shapes for drag and swipe gestures were observed. The vectors for horizontal gestures are slightly tilted upwards towards the middle of the screen, comparable to a fishbone pattern. Vertical gesture paths are drawn like a bow, and the imaginary middle of the circle would be inside the palm (Figure 2)

Gesture orientation. The gesture path orientations (horizontal, vertical) had no influence on the vector parameters (gesture path length: $F_{1,953}=2.379$, $p=0.123$, gesture execution duration: $F_{1,1623}=1.545$, $p=0.214)$ in an ANOVA.

\begin{tabular}{|c|c|c|c|c|c|}
\hline \multicolumn{2}{|c|}{$\begin{array}{l}\text { Path length in px } \\
\quad \text { (in mm) }\end{array}$} & \multicolumn{2}{|c|}{ Duration in s } & \multicolumn{2}{|c|}{ Velocity in px/s } \\
\hline left ${ }^{s m}$ & right ${ }^{N M y}$ & left ${ }^{\mathrm{s} y}$ & right ${ }^{\mathrm{s} m}$ & left & right ${ }^{3} y$ \\
\hline \multicolumn{6}{|c|}{ Tap on the touchscreen } \\
\hline 1 & 1 & 0.2 & 0.2 & 5 & 5 \\
\hline \multicolumn{6}{|c|}{ Press on the touchscreen } \\
\hline 4 & 7 & 0.7 & 0.7 & 6 & 10 \\
\hline \multicolumn{6}{|c|}{ Vertical drag on the touchscreen } \\
\hline $314(48)$ & (291) 45 & 1.0 & 1.1 & 249 & 251 \\
\hline \multicolumn{6}{|c|}{ Horizontal drag on the touchscreen } \\
\hline $247(38)$ & $221(34)$ & 0.8 & 0.7 & 216 & 233 \\
\hline \multicolumn{6}{|c|}{ Vertical swipe on the touchscreen } \\
\hline $265(41)$ & $285(44)$ & 0.3 & 0.2 & 10757 & 10689 \\
\hline \multicolumn{6}{|c|}{ Horizontal swipe on the touchscreen } \\
\hline $212(32)$ & $214(33)$ & 0.2 & 0.2 & 12188 & 15678 \\
\hline \multicolumn{6}{|c|}{ Tap on the back of the tablet } \\
\hline 4 & 8 & 0.2 & 0.2 & 20 & 40 \\
\hline \multicolumn{6}{|c|}{ Press on the back of the tablet } \\
\hline 17 & 18 & 0.7 & 0.7 & 24 & 26 \\
\hline \multicolumn{6}{|c|}{ Vertical drag on the back of the tablet } \\
\hline $213(32)$ & $183(28)$ & 0.9 & 0.7 & 2586 & 2569 \\
\hline \multicolumn{6}{|c|}{ Horizontal drag on the back of the tablet } \\
\hline $206(32)$ & $193(30)$ & 0.9 & 0.7 & 2632 & 2560 \\
\hline \multicolumn{6}{|c|}{ Vertical swipe on the back of the tablet } \\
\hline $196(30)$ & $203(31)$ & 0.3 & 0.3 & 2926 & 2617 \\
\hline \multicolumn{6}{|c|}{ Horizontal swipe on the back of the tablet } \\
\hline $189(29)$ & $174(27)$ & 0.3 & 0.2 & 3211 & 2700 \\
\hline
\end{tabular}

Table 1. Typical gestures: medians for path length (Figure 2), gesture duration, \& velocity on both device sides, both gesture orientations $\&$ with both hands. 
Hand symmetry. The gesture execution is symmetric for both hands as shown in Figure 2. This means that the gesture vectors are flipped on an imaginary vertical line drawn in the middle of the screen. Thus, the positions (considering that they are vertically flipped) are not affected $(p=0.929)$, and the drag as well as swipe paths do not differ in length regardless of whether they have been executed with left or right hand $(p=0.885)$. While the gesture vectors look similar for the right or left hand, gesture execution duration differs significantly for both hands. Moreover, a significant interaction effect between hand and gesture was found for execution duration $(p=0.003)$.

Front versus back of the device. The gestures differ in path length if they were executed on the front versus on the back of tablet $(p>0.001)$. Moreover, Figure 2 shows that the gesture vectors of drag and swipe are significantly longer than tap and press, which by definition are close to zero. Swipe and tap are faster than drag and press $(p>0.001)$. Their values are almost independent and can therefore serve as gesture classification as it is already the case in common touch gesture APIs like for iOS, Android, and mobile browsers. Interestingly, the duration and path length sometimes vary for the same gesture when executed on different device sides: drag and press are slightly, but significantly longer on the front side where the gestures are performed with the thumb. Moreover, the vectors for drag and swipe are a significantly longer when performed with the thumb on the front of the tablet compared to the gesture paths on the back side. Thus, an interaction effect was found for gesture* device side (Figure 3 ) and swipe on the back of the device has much less velocity $(p>0.001)$ than on the front.

\section{Discussion \& conclusion}

The gestures were executed close to the positions where the hands were grasping the device. These positions are in line with the interaction areas for bimanual held tablets defined for touchscreens by Odell and Chandrasekaran [4]. The parameters of the gesture paths are significantly influenced by the gesture type and the device side. The used hand influenced execution time but not the paths. The gesture orientation was neither affecting execution time nor path.

On mobile devices, gesture recognition thresholds are defined by these parameters for front- but not for backof-device interaction. In the following section, we compare our parameters with common gesture recognition parameters of Android APIs, e.g. accessible through the getLongPressTimeout command [1].

Android versus observed parameters

- Our durations of tap (0.2s) and press (0.7s) for both, the front and the back of the device are in line with the Android thresholds (tap: <0.5s, press: $>0.5 \mathrm{~s}$ ).

- Our prototypical drag performed on a tablet is 30 to $50 \mathrm{~mm}$. Such values do not exist for (Android and others') gesture detection, but they help designing widgets, such as defining slider length.

- Our detected velocity for swiping on the frontscreen (avrg_front: vertical: 10723, horizontal: 13933; avrg_back: horizontal: 2956, vertical: 2772), which serves for mapping the swipe execution onto scroll friction, is within and for horizontal front swipe above the range of several Android phones (from 75 to 12000 pixels per second) and for back-of-device 
within, but for touchscreen interaction above the range of the Android ASUS tablet (between 50 and 8000 pixels per seconds). We rate values above the common ranges as acceptable: users would just have no problems to scroll fast. If the maximum velocity cannot be reached, which is suggested for back-of-device interaction, the mapping should be defined correspondingly. As back-of-device swiping was up to four times smaller for back-of-tablet interactions compared to swipe velocity on the front, dividing scroll parameters by four for back-of-device interaction would be recommended.

- No thresholds are defined in existing APIs for distinguishing an unintended touch event. That event would cause Midas touch problems in back-ofdevice interaction. According to the presented results, touch events on the back of tablets that last longer than $1.0 \mathrm{sec}$ (considering 1 SD safety distance from the press/taphold mean) without moving are unintended touch events.

In summary, gestures on the front take longer (drag, press) and are drawn over a greater distance (drag, swipe) than gestures on the back of the device. A reason for this is probably that gestures on the front are executed with the thumb is more flexible than the fingers [7]. Thus, design guidelines for touchscreen interaction cannot automatically be adapted for backof-device interaction, and parameters that should to be defined differently for front versus back-of-device interaction are suggested.

\section{References}

[1] Android Developers Reference: developer.android.com/reference/android/view/ ViewConfiguration.html, 27.7.2013.
[2] Hiraoka, S., Miyamoto, I. and Tomimatsu, K. 2003. Behind Touch: A text input method for mobile phone by the back and tactile sense interface. Proc. Interaction 2003. Information Processing Society of Japan, 131138.

[3] Hoggan, E., Williamson, J., Oulasvirta, A., Nacenta, M., Kristensson, P.O., Lehtiö, A. 2013. Multi-touch rotation gestures: performance and ergonomics. In Proc. CHI '13, 3047-3050.

[4] Odell, D. and Chandrasekaran, V. 2012. Enabling comfortable thumb interaction in tablet computers: a Windows 8 case study. In Proc. of the Human Factors and Ergonomics Society Annual Meeting, 1907-1911.

[5] Ohtani, T., Hashida, T., Kakehi, Y., and Naemura, T. 2011. Comparison of front touch and back touch while using transparent double-sided touch display. In ACM SIGGRAPH 2011 Posters (SIGGRAPH '11).

[6] Oulasvirta, A., Reichel, A., Li, W., Zhang, Y., Bachynskyi, M., Vertanen, K., Kristensson, P.O. 2013. Improving two-thumb text entry on touchscreen devices. In Proc. CHI '13, 2765-2774.

[7] Vardy, A. 1998. Articulated Human Hand Model with Inter-Joint Dependency Constraints. Computer Science 6752, Comp. Graphics, Project Report, 1-13.

[8] Wagner, J., Huot, S., and Mackay, W.E. 2012. BiTouch and BiPad: designing bimanual interaction for hand-held tablets. In Proc. CHI'12, 2317-2326.

[9] Wigdor, D., Forlines, C., Baudisch, P., Barnwell, J., and Shen, C. 2007. Lucid touch: a see-through mobile device. In Proc. UIST'07, 269-278.

[10] Wobbrock, J.O., Myers, B.A., Aung, H.H. 2008. The performance of hand postures in front- and back-ofdevice interaction for mobile computing. International Journal of Human-Computer Studies 66 (12), 857-875.

[11] Wolf, K., Müller-Tomfelde, C., Cheng, K., and Wechsung, I. 2012. Does proprioception guide back-ofdevice pointing as well as vision? In EA CHI'12, 17391744. 